\title{
Neutron-captures in Low Mass Stars and the Early Solar System Record of Short-lived Radioactivities
}

\author{
Maurizio Busso ${ }^{1,2, \star}$, Diego Vescovi ${ }^{1,2}$, Oscar Trippella ${ }^{1,2}$, Sara Palmerini ${ }^{1,2}$, Sergio Cristallo ${ }^{3,2}$, \\ and Luciano Piersanti ${ }^{3,2}$ \\ ${ }^{1}$ Department of Physics and Geology, Via A. Pascoli, 06123 Perugia, Italy \\ ${ }^{2}$ I.N.F.N., Section of Perugia, Via A. Pascoli, 06123 Perugia, Italy \\ ${ }^{3}$ I.N.A.F., Observatory of Abruzzo, Teramo, Italy
}

\begin{abstract}
Noticeable improvements were recently introduced in the modelling of $\mathrm{n}$ capture nucleosynthesis in the advanced evolutionary stages of giant stars (Asymptotic Giant Branch, or AGB, stars). Two such improvements are closely linked together and concern the introduction of non-parameterized, physical models for extended mixing processes and the adoption of accurate reaction rates for $\mathrm{H}$ - and He-burning reactions, including the one for the main neutron source ${ }^{13} \mathrm{C}(\alpha, \mathrm{n}){ }^{16} \mathrm{O}$. These improvements profited of a longstanding collaboration between stellar physicists and C. Spitaleri's team and of his seminal work both as a leader in the Nuclear Astrophysics scenario and as a talentscout in the recruitment of young researchers in the field. We present an example of the innovative results that can be obtained thanks to the novelties introduced, by estimating the contributions from a nearby AGB star to the synthesis of short-lived $\left(t_{1 / 2} \leq 10 \mathrm{Myr}\right)$ radioactive nuclei which were alive in early Solar System condensates. We find that the scenario indicating an AGB star as the source of such radioactivities, discussed for many years by researchers in this field, appears now to be no longer viable, when the mentioned improvements of AGB models and nuclear parameters are considered.
\end{abstract}

\section{Introduction}

Computations of slow neutron capture nucleosynthesis (the so-called $s$-processing) in the final evolutionary phases of red giant stars (the AGB stages) were for many years based on parameterized approaches. In particular, several years ago it was recognized [1-3] that the main neutron source had to be identified with the ${ }^{13} \mathrm{C}(\alpha, \mathrm{n}){ }^{16} \mathrm{O}$ reaction, starting from an abundance of ${ }^{13} \mathrm{C}$ necessarily much larger than the equilibrium concentration left for this nucleus by the occurrence of H-burning in a shell. Fresh ${ }^{13} \mathrm{C}$ had to be synthesized locally; its abundance was usually varied parametrically $[3,4]$ in order to derive constraints on its production from observations. This last was suggested to occur during the recurrent inward extensions of the envelope, known collectively under the name of Third Dredge-up or TDU. Even in absence of a model, it was assumed that at TDU deep mixing phenomena could inject protons into the He-rich layer in concentrations suitable to interact with the abundant ${ }^{12} \mathrm{C}$, producing the required ${ }^{13} \mathrm{C}$.

\footnotetext{
^e-mail: maurizio.busso@pg.infn.it
} 
Today we deal with a scenario noticeably improved with respect to those early attempts. The upgrades would not have been possible, if not for the longstanding collaboration in Nuclear Astrophysics issues between research groups studying stellar physics and the team founded and headed by Claudio Spitaleri, who provided for many years a wealth of new accurate estimates for crucial reaction rates obtained by the ASFIN collaboration through the indirect experimental method known as Trojan Horse. He also helped in various ways in the recruitment of bright young researchers in the field. In this last role he has become known worldwide especilly for his Santa Tecla school, one of the most successful and rewarding initiatives in the whole Nuclear Astrophysics panorama.

Among the rates which were provided by Claudio and his collaborators, and that are now adopted in our nucleosynthesis computations, we remind first of all the one for $(\alpha, n)$ captures on ${ }^{13} \mathrm{C}$, i.e. the neutron source itself $[5,6]$. Moreover, of high relevance are the cross sections for p-induced reactions on intermediate-mass isotopes, which must be considered as they hamper the possibility of those nuclei to capture neutrons or $\alpha$ particles. Among them we remind ( $\mathrm{p}, \alpha)$ reactions on ${ }^{15} \mathrm{~N}[7]$, on ${ }^{17} \mathrm{O},{ }^{18} \mathrm{O}[8,9]$ and on ${ }^{19} \mathrm{~F}[10,11]$. More recently, ASFIN made available also the rate for $(\alpha, \mathrm{p})$ captures on ${ }^{19} \mathrm{~F}$, occurring during the thermal instabilities of the He-shell in direct competition with both $(\alpha, \gamma)$ and $(\alpha, \mathrm{n})$ captures on ${ }^{18} \mathrm{O}$ and ${ }^{22} \mathrm{Ne}$ [12]. Finally, neutron captures have started to become available from the collaboration, see e.g. the case of ${ }^{17} \mathrm{O}(\mathrm{n}, \alpha){ }^{14} \mathrm{C}$ [13], which is important for the neutron absorption from the daugther nucleus ${ }^{14} \mathrm{~N}$.

Another crucial ingredient, as important as the availability of high-quality nuclear physics inputs, was the formulation of physical models for the non-convective mixing processes affecting the inner border of the convective envelope in AGB stars.

Recently, models for deep mixing phenomena have been explored by various groups in the aim of overcoming the uncertain parameterizations and provide a more quantitative knowledge of AGB nucleosynthesis. Among such attempts, we recall the work by [14], exploring the effects of gravity waves and Kelvin-Helmoltz instabilities. This work revisited previous suggestions by $[15,16]$ and others. Another approach was pursued by $[17,18]$, on the basis of revisions of the physics at the convective border; similar attempts were also made by [19]. Concerning the study of low-metallicity AGB stars and the peculiar coupling of proton injection and nucleosynthesis occurring in them, see also [20].

A rather different paradigm was discussed by [21,22] and by [23]. In it, an extended penetration of protons into the He-rich layers is induced as a byproduct of Magneto-hydrodynamical (MHD) effects, namely the buoyancy of magnetized bubbles, formed deep below the TDU inner border and forced to emerge into the envelope by their extra pressure provided by the magnetic field. This buoyancy pushes down protons for mass conservation: see details of the original MHD simulations in [24, 25] and, especially, [26].

Although it is still premature to judge which one of the above-mentioned suggestions will prove to be more promising, they all point to the same direction, i.e. the need of overcoming the era of free parameterizations, passing to more physical types of modelling. Then the complexity of stellar plasma phenomena makes it probable that more than one mechanism be at play in AGB stars.

In this contribution we shall in particular consider the approach by [22] that was already shown to be able to produce extended ${ }^{13} \mathrm{C}$ reservoirs and to induce neutron captures capable of reproducing not only the solar-system distribution of $s$-elements, but also the more stringent constraints [27-29] that are posed by the isotopic composition of presolar SiC grains: see e.g. [30]. As an example of the further results that can be obtained in this scenario, we consider here the possible contributions of an intermediate-mass star to the production of the short-lived ( $\tau \leq 20 \mathrm{Myr}$ ) radioactivities (SLR) that were found to be alive in the first condensates of the Early Solar System (ESS). The complementary mixing scenario suggested by $[17,18]$ will be considered in a forthcoming paper. 
For computing the detailed nucleosynthesis pattern produced by an AGB star we had to adopt an extended set of cross sections for $p$ - and $\alpha$-capture reactions on light and intermediate nuclei; this includes many more processes, in addition to those mentioned above. They were taken from the compilation by [31], having again Claudio Spitaleri as a coauthor. Concerning the neutron captures induced by the $(\alpha, n)$ neutron sources active in the He-layers of an AGB star, we followed the recommendations by [32] and [33]. Initial abundances were chosen to be solar, and were adopted from [34].

\section{An Attempt to Explain ESS Radioactivites with an IMS Star.}

Starting from the pioneering work by [35], suggesting the presence of live ${ }^{129} \mathrm{I}$ in pristine Solar System materials, the inventory of radioactive nuclei demonstrated to be alive in the ESS grew over the years considerably. Recent discussions of the present-day ideas on the early concentrations of those nuclei were presented by [36] and by [37]. The emerging situation is summarized in Table 1 (see the quoted papers for the original experimental works on which the Table is based).

Table 1. Short-Lived Nuclei in the ESS

\begin{tabular}{|c|c|c|c|}
\hline Rad. & Ref. & $\begin{array}{c}\text { Mean Life } \\
(\mathrm{Myr})\end{array}$ & $\begin{array}{c}\left(N_{P} / N_{I}\right)_{\text {ESS }} \\
\text { at } t=0\end{array}$ \\
\hline${ }^{10} \mathrm{Be}$ & ${ }^{9} \mathrm{Be}$ & 2.0 & $\sim 5 \cdot 10^{-4}$ \\
${ }^{26} \mathrm{Al}$ & ${ }^{27} \mathrm{Al}$ & 1.03 & $(5-5.2) \cdot 10^{-5}$ \\
${ }^{36} \mathrm{Cl}$ & ${ }^{35} \mathrm{Cl}$ & 0.43 & $10^{-4}$ \\
${ }^{41} \mathrm{Ca}$ & ${ }^{40} \mathrm{Ca}$ & 0.15 & $\geq 1.5 \cdot 10^{-8}$ \\
${ }^{53} \mathrm{Mn}$ & ${ }^{55} \mathrm{Mn}$ & 5.3 & $6.7 \cdot 10^{-5}$ \\
${ }^{60} \mathrm{Fe}$ & ${ }^{56} \mathrm{Fe}$ & 3.75 & $(2-4) \times 10^{-7}$ \\
${ }^{107} \mathrm{Pd}$ & ${ }^{108} \mathrm{Pd}$ & 9.4 & $5.9( \pm 2.2) \cdot 10^{-5}$ \\
${ }^{129} \mathrm{I}$ & ${ }^{127} \mathrm{I}$ & 23 & $1.0 \cdot 10^{-4}$ \\
${ }^{135} \mathrm{Cs}$ & ${ }^{133} \mathrm{Cs}$ & 3,3 & $(1-2) \cdot 10^{-4}$ \\
${ }^{146} \mathrm{Sm}$ & ${ }^{144} \mathrm{Sm}$ & 148 & $1.0 \cdot 10^{-2}$ \\
${ }^{182} \mathrm{Hf}$ & ${ }^{180} \mathrm{Hf}$ & 12.8 & $9.7 \cdot 10^{-5}$ \\
${ }^{205} \mathrm{~Pb}$ & ${ }^{204} \mathrm{~Pb}$ & 22 & $(1-2) \cdot 10^{-4}$ \\
${ }^{244} \mathrm{Pu}$ & ${ }^{232} \mathrm{Th}$ & 115 & $3 \cdot 10^{-3}$ \\
${ }^{247} \mathrm{Cm}$ & ${ }^{232} \mathrm{Th}$ & 23 & $(1-2.4) \cdot 10^{-4}$ \\
\hline
\end{tabular}

We adopted the model of a $5 \mathrm{M}_{\odot}$ star of solar metallicity from the calculations of full stellar models performed with the Schwarzschild criterion for convection, as part of the input material for the review by [38]. The star has a lifetime slightly longer than $100 \mathrm{Myr}$ and was computed with a Reimers-like mass loss, using the value $\eta=3$ for the free parameter of the formula. Figure 1 illustrates a sketch of the evolution in time of the stellar structure; there the $\mathrm{C}-\mathrm{O}$ core, the He-rich buffer and the overlying convective envelope with its recurrent inward extensions (TDU) are shown. The H-rich buffer below the convective envelope is barely appreciable as its mass is smaller than the one of the He-rich zone by more than a factor of 10 .

Inspection of the physical structure of the model reveals that conditions suitable for obtaining the analytical solution of MHD equations discussed by [26] exist in two thin layers sited, respectively, above the H-burning shell and below the inner border of convection at TDU. These conditions include a density that drops rapidly as a negative power-law of the radius, a temperature that decreases more 


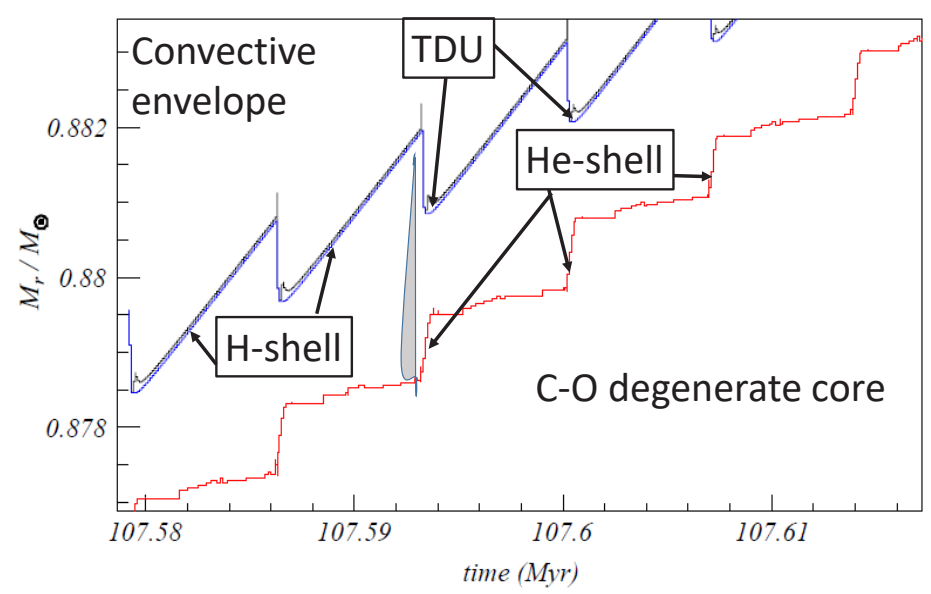

Figure 1. The basic structure of our model star during the thermally pulsing AGB phase around the 5th thermal pulse (shaded in grey). Note how thin is the H-rich layer below the convective envelope.

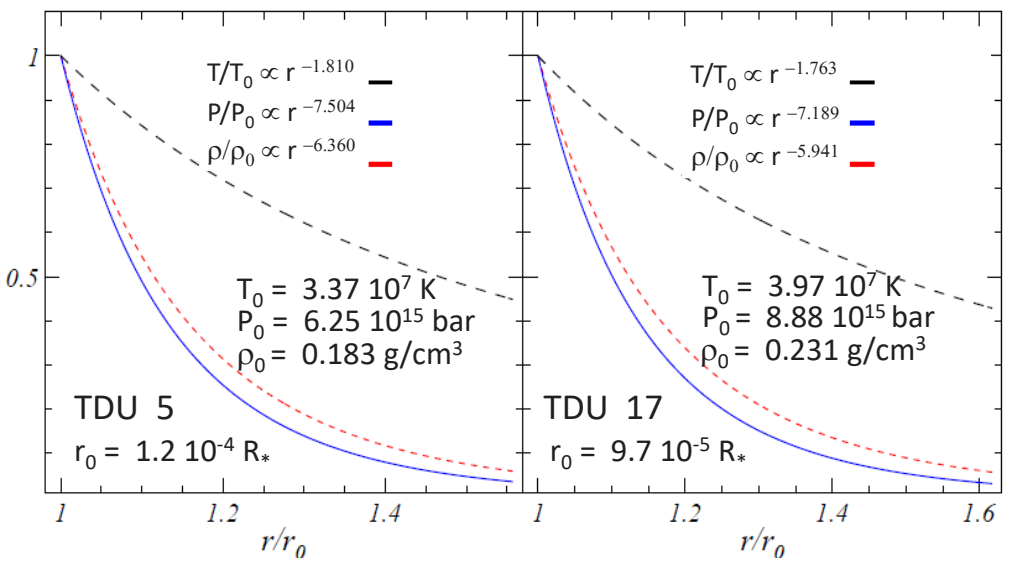

Figure 2. The physical conditions, in terms of temperature, density and pressure, of the layers in the He-rich zone where a quasi-ideal MHD condition holds, so that one expects that a circulation of material occurs for magnetic buoyancy, inducing the penetration of protons from the envelope and the formation of a ${ }^{13} \mathrm{C}$ pocket. The mass of such a pocket is smaller than for a star of $1.5 \mathrm{M}_{\odot}$ by a factor from 28 to 35 , depending on the pulse number.

rapidly than $1 / r$, a small kinematic viscosity and a large value for the magnetic Prandtl number. These conditions imply that magnetic buoyancy easily induces mixing there. Above the H-burning shell this yields the synthesis of some ${ }^{26} \mathrm{Al}$, but this production is not very efficient, due to the thin radiative $\mathrm{H}$-rich layer. In the He-zone, a ${ }^{13} \mathrm{C}$ pocket is formed. This last is established over a very small mass 
region (smaller by some 30 times than in low mass stars). Nevertheless, some neutron captures do occur in such a pocket, adding their effects to the $s$-processing induced by the ${ }^{22} \mathrm{Ne}$ source [2] in the convective region before a TDU episode. We therefore find that a condition like the one advocated by [39], in which an AGB star was assumed to host extramixing phenomena in the radiative H-rich zone, without the formation of a ${ }^{13} \mathrm{C}$ pocket, cannot occur.

This fact is fatal for the possibility of an AGB star (of any mass) to explain the ESS radioactivities. Indeed, ${ }^{26} \mathrm{Al}$ is produced only moderately by deep mixing processes and any ${ }^{13} \mathrm{C}$ burning in the Helayers invariably leads to a ratio ${ }^{107} \mathrm{Pd} /{ }^{26} \mathrm{Al}$ by far too high than required to account for the extinct radioactivities. Furthermore, the limited number of neutrons available from ${ }^{13} \mathrm{C}$ burning strongly favors $\mathrm{Pd}$ and $\mathrm{Cs}$ over $\mathrm{Hf}$ and $\mathrm{Pb}$; also the production ${ }^{60} \mathrm{Fe}$ is excessive and a global solution is impossible.

An example of such finding is shown in Table 2, where we applied, to the envelope composition at the end of the evolution of our model star, the procedure described in $[36,37,39]$ to infer the concentrations of some radioctive nuclei in a forming star close in time and space to the mass losing $5 \mathrm{M}_{\odot}$ AGB star we modelled. As the Table shows, if we try to fit $\mathrm{Al}$ and $\mathrm{Ca}$ all the other nuclei are not explained.

TABLE 2.

Our Predictions for Short Lived Nuclei in the ESS.

\begin{tabular}{|c|c|c|c|}
\hline & $5.0 \mathrm{M}_{\odot}$ & $\begin{array}{c}\mathrm{Z}=Z_{\odot} \\
f_{0}=2.56 \times 10^{-2}\end{array}$ & $\begin{array}{c}\text { Measured } \\
\text { or extrapolated }\end{array}$ \\
\hline $\begin{array}{c}\text { Parent } \\
P\end{array}$ & $\begin{array}{c}\text { Index } \\
I\end{array}$ & $\begin{array}{c}\left(N_{P} / N_{I}\right)_{\Delta_{1}} \\
\Delta_{1}=0.65 \mathrm{Myr}\end{array}$ & at $t=\Delta_{1}$ \\
\hline${ }^{26} \mathrm{Al}$ & ${ }^{27} \mathrm{Al}$ & $5.05 \cdot 10^{-5}$ & $(5-5.2) \cdot 10^{-5}$ \\
\hline${ }^{41} \mathrm{Ca}$ & ${ }^{40} \mathrm{Ca}$ & $1.08 \cdot 10^{-8}$ & $\geq 1.5 \cdot 10^{-8}$ \\
\hline${ }^{60} \mathrm{Fe}$ & ${ }^{56} \mathrm{Fe}$ & $1.15 \cdot 10^{-5}$ & $(2-4) \cdot 10^{-7}$ \\
\hline${ }^{107} \mathrm{Pd}$ & ${ }^{108} \mathrm{Pd}$ & $8.1 \cdot 10^{-4}$ & $5.9( \pm 2.2) \cdot 10^{-5}$ \\
\hline${ }^{135} \mathrm{Cs}$ & ${ }^{133} \mathrm{Cs}$ & $7.6 \cdot 10^{-4}$ & $(1-2) \cdot 10^{-4}$ \\
\hline${ }^{182} \mathrm{Hf}$ & ${ }^{180} \mathrm{Hf}$ & $1.24 \cdot 10^{-5}$ & $9.7 \cdot 10^{-5}$ \\
\hline${ }^{205} \mathrm{~Pb}$ & ${ }^{204} \mathrm{~Pb}$ & $1.89 \cdot 10^{-6}$ & $(1-2) \cdot 10^{-4}$ \\
\hline
\end{tabular}

We remind that recently [36] failed to explain the ESS radioactivities, even considering more massive AGB stars, producing ${ }^{26} \mathrm{Al}$ from Hot Botton Burning. If those results and our ones will be confirmed, then this would put an end to the idea that a chance encounter with an AGB star might be at the origin of the isotopic anomalies of pristine meteorites induced by the decay of short lived radioactivities. If this were the case, then the most promising alternatives might reside either in the sequential pollution of the parent molecular cloud by various massive stars, as in the model by [40] or in some revision of a single SN contributions, e.g. in the new scenarios of rotating massive stars [41].

\section{References}

[1] Iben, I., Jr. and Renzini, A. 1983, ARA\&A 21, 271

[2] Busso, M., Gallino, R., Wasserburg, G.J. 2001, ARAA 37, 239

[3] Gallino, R., Arlandini, C., Busso, M., et al. 1998, ApJ 497, 388

[4] Käppeler, F., Gallino, R., Bisterzo, S. et al. 2011, Rev. Mod. Phys. 83, 157

[5] La Cognata, M., Spitaleri, C., Trippella, O., et al. 2013, ApJ 777, 143 
[6] Trippella, O., La Cognata, M. 2017, ApJ 845, 191

[7] La Cognata, M., Goldberg, V.Z., Mukhamedzhanov, A.M. et al. 2009, Phys. Rev. C 80, 12801

[8] La Cognata, M., Spitaleri, C., Mukhamedzhanov, A.M, et al. 2010 ApJ 708, 796

[9] Palmerini, S., Sergi, M.L., La Cognata, M. et al. 2013, ApJ 764, 128

[10] La Cognata, M., Mukhamedzhanov, A.M., Spitaleri, C., et al. 2011, ApJ 739, L54

[11] Indelicato, I., La Cognata, M., Spitaleri, C., et al. 2017, ApJ 845, 191

[12] Pizzone, R.G., D’Agata, G., La Cognata, M., et al. 2017, ApJ 836, 57

[13] Guardo, G., Spitaleri, C., Lamia, L. et al. 2017, Phys. Rev. C. 95, 25807

[14] Battino, U., Pignatari, M., Ritter, C., et al. 2016, ApJ 827, 30

[15] Denissenkov, P.A., and Tout, C.A. 2003, MNRAS 340, 322

[16] Talon, S. 2008, in EAS Publication Series vol 32, ed. C. Chabonnel and J.-P. Zahn, p. 81

[17] Cristallo, S., Piersanti, L., Straniero, O., et al. 2011, ApJS 197, 17

[18] Cristallo, S., Straniero, O., Piersanti, L., Gobrecht, D. 2015 ApJS 219, 40

[19] Pignatari,. M., Herwig, F., Hirschi, R. et al. 2016, ApJS 225, 24

[20] Stancliffe, R.J., Dearborn, D.S.P., Lattanzio, J.C., et al. 2011, ApJ 742, 2

[21] Trippella, O., Busso, M., Maiorca, E., et al. 2014, ApJ 787, 41

[22] Trippella, O., Busso, M., Palmerini, S., et al. 2016, ApJ 818, 125

[23] Palmerini, S., Trippella, O., and Busso, M., 2017a, MNRAS 467, 1193

[24] Busso, M., Wasserburg, G.J., Nollett, K.M., and Calandra, A. 2007, ApJ 671, 802

[25] Nordhaus, J., Busso, M., Wasserburg, G.J., Blackman, E.G., and Palmerini, S. 2008, ApJ 684, L29

[26] Nucci, M.C., and Busso, M. ApJ 2014, 787, 141

[27] Liu, N., Gallino, R., Bisterzo, S., et al. 2014a, ApJ 786, 66

[28] Liu, N., Gallino, R., Bisterzo, S., et al. 2014b, ApJ 788, 163

[29] Liu, N., Savina, M.R., Gallino, R. et al. 2015, ApJ 803, 12

[30] Palmerini, S., Trippella, O., Busso, M., Vescovi, D., et al. 2017b, GCA in press.

[31] Adelberger, E. G., García, A., Robertson, R. G., et al. 2011, Rev. Mod. Phys. 83, 195.

[32] Dillmann, I., Heil, M., Käppeler, F., et al., 2006, AIP Conference Proceedings 819, 123

[33] Dillmann, I. 2014, in Proceedings of the XIII Symposium Nuclei in the Cosmos, http://pos.sissa.it/cgi-bin/reader/conf.cgi?confid=204, id.57

[34] Lodders, K. and Palme, H. 2009, MPSS, 72, 5154

[35] Reynolds, J. H. 1960, Phys. Rev. Lett. 4, 351.

[36] Wasserburg, G. J., Karakas, A. I, and Lugaro, M. 2017, ApJ 836, 126

[37] Busso, M. 2018, in Astronomy with Radioactivities (Springer: in press)

[38] Straniero, O., Domínguez, I., Cristallo, S., Gallino, R. 2003, PASA 20, 389

[39] Wasserburg, G. J., Busso, M., Gallino, R., Nollett, K. M. 2006, Nucl. Phys. A, 777, 5

[40] Gounelle, M. and Meynet, G. 2012, A\&A 545, 4

[41] Limongi, M, \& Chieffi, A. 2015, in Chemical and dynamical evolution of the Milky Way and the Local Group, Proc. Sedem Conf. id 15 